Fecha de recepción: diciembre 2018 Fecha de aceptación: marzo 2019 Versión final: abril 2019

\section{Entre la forma de habitar y las formas para habitar. Vivienda campesina y arquitectura vernácula: nociones morfológicas}

Genoveva Malo *

Resumen: Este texto propone el análisis de los referentes y condiciones que configuran la vivienda popular campesina en las provincias ecuatorianas de Azuay y Cañar, como expresión y forma de la arquitectura vernácula, a partir problematizar el sistema del habitar rural. Se describen y analizan configuraciones espaciales en el marco de un esquema conceptual que toma al contexto como nodo articulador de sentido y análisis, a partir de reflexiones de Jorge Pokropek (2020), quien plantea la relación entre prácticas sociales y las configuraciones espaciales portadoras de sentido e identidad para definir lógicas de coherencia como mecanismo de interpretación y producción de diseño interior, así como la noción de forma propuesta por Dora Giordano (2018) como producto cultural, y Joseph María Montaner quien plantea comprender a la forma como una estructura, más allá de la configuración geométrica. Las reflexiones y análisis sobre el tema que se expone se sustentan en datos obtenidos en una investigación realizada entre la Universidad de Cuenca y la Universidad del Azuay en 2016 que toma datos y dibujos a mano (plumillas y fichas de observación) de la arquitectura vernácula entre 1977 y 1978 en las provincias de Azuay y Cañar.

Palabras clave: Forma del habitar - noción de forma - morfología de la vivienda - vivienda campesina - arquitectura vernácula

[Resúmenes en inglés y portugués en las páginas 100-101]

Afiliación institucional: Universidad del Azuay, Cuenca-Ecuador.

${ }^{(*)}$ Doctoranda en Diseño por la Universidad de Palermo. Diseñadora por la Facultad de diseño de la Universidad del Azuay (1996), Master en Diseño por la misma Universidad (2009) Diplomada en Gerencia Estratégica de Mercado por la Universidad Técnica Particular de Loja (2007)

\title{
Introducción
}

La vivienda campesina, como una de las expresiones de la arquitectura vernácula, es el reflejo de modos de vida y prácticas que devienen en configuraciones espaciales con sorprendentes ejemplos de eficacia constructiva, integración al entorno, máxima simplicidad, 
humildad, sencillez expresiva y funcional como reflejo de una clara conexión con el entorno y respuesta a las necesidades planteadas en un contexto determinado. El estudio de estas formas (morfología), así como las formas de vida (formas de habitar), constituye, sin duda, una oportunidad para el análisis y transferencia de conceptos morfológicos para el interiorismo y arquitectura contemporánea.

El concepto de arquitectura vernácula no es estático, sino que ha evolucionado a través del tiempo, desde concepciones asociadas a condiciones de pobreza y poca valoración, con referencias a construcciones originarias del lugar, que estaban presentes en las narrativas de los viajeros y colonizadores (Zorrila, 2015) hasta importantes reconocimientos en el campo de la cultura y la arquitectura hacia los años sesentas en las voces de algunos expertos como Rappoport (1969) quien reivindica el valor cultural de este tipo de arquitectura, alejándola del concepto de subdesarrollo y nostalgia.

Rappoport (1969) hace un primer acercamiento a la noción contemporánea de arquitectura vernácula en términos de "aquella en la que no existen pretensiones teóricas o estéticas; que trabaja con el lugar de emplazamiento y con el microclima; respeta las demás personas y sus casas y, en consecuencia, al ambiente total, natural o fabricado por el hombre y trabaja de un idioma con variaciones dentro de un orden dado" (pág. 12). Precisamente en el marco de esta definición nos interesa ahondar en la concepción de una morfología de la vivienda que entabla relaciones con el entorno (espacio exterior) y con los modos habitar (espacio interior) bajo una noción de habitabilidad.

Si bien no existe una definición consensuada para la arquitectura vernácula, la que interesa para esta reflexión es aquella propuesta por el Consejo Internacional de Monumentos y Sitios - ICOMOS (1999) y que en un sentido general habla de "un proceso continuo, que incluye cambios necesarios y una continua adaptación como respuesta a los requerimientos sociales y ambientales".Es decir, una forma de arquitectura fuertemente marcada por un entorno.

Se propone así, una reflexión y análisis de la forma de este tipo o tipos de vivienda y su configuración espacial como objeto y como espacio, a la luz de teorías morfológicas y de noción de forma expuestas por Jorge Pokropek (2020), Dora Giordano (2018) y Joseph María Montaner (2002).

Las interpretaciones se abordan desde un enfoque hermenéutico que no cierra la reflexión sino, por el contrario, abre caminos hacia otros procesos de construcción de significados que no es unívoca. Se comprende, para este análisis, a la vivienda campesina vernácula, como forma en cuanto establece relaciones espaciales externas, con su entorno, e internas con una configuración espacial (interiorismo) vinculadas a la cultura y modos de habitar. La elección, para este análisis morfológico, de autores populares anónimos de la provincia del Azuay y Cañar en Ecuador que han construido por generaciones viviendas populares campesinas, responde al interés por conocer y re conocer la relación de las configuraciones espaciales con las condicionantes y/o referentes del contexto y la prácticas de vida campesina; así como los modos en que éstas determinan modos/formas de habitar y las formas/espacios para habitar. Estas reflexiones podrían aportar al conocimiento sobre la morfología de la vivienda y su relación con el contexto en las diversas prácticas sociales. Una hipótesis provisoria plantearía, para esta reflexión, que la morfología de la vivienda 
popular campesina, como expresión de la arquitectura vernácula muestra signos de una fuerte coherencia con el entorno y las prácticas sociales.

Entre los aspectos morfológicos que destaca este análisis están: la configuración espacial, los emplazamientos, la distribución, el lenguaje formal y los detalles constructivos como elementos de alta significación. Interesa profundizar en la relación habitabilidad/morfología en relación a la cultura, en especial, la cultura popular.

\section{Marco Teórico}

Pokropek (2020) plantea que "nuestras prácticas sociales exigen para su eficaz desempeño la configuración de espacialidades o ambientaciones estéticamente intencionadas que tiendan a planificar la experiencia humana al expresar lógicas de coherencia entre las diversas formas objetuales necesarias para dichas prácticas y los entornos en donde deben ubicarse" (pág. 19), aseveración que podría generalizarse a la mayoría de casos en la experiencia contemporánea de uso de espacios/objetos habitables, y que resulta interesante revisar cómo se configura un tipo de vivienda particular: la vivienda popular campesina que muestra fuertes signos de coherencia con el entorno y las prácticas sociales.

Para orientar en lo que el autor llama las "lógicas de coherencia", propone algunos caminos de reflexión y producción de formas:

Las categorías estructurales (formas arquitectónicas y estructuras abstractas para establecer lógicas de configuración y lógicas de organización),

Las categorías texturales (expresión de la materialidad para establecer relaciones forma-conducta, forma-emoción) y

Las cadenas armónicas como la expresión de polaridades puestas en juego, así como en los tipos configurativos espaciales básicos y las respuestas conductoemocionales que éstos promueven (Pokropek, 2020).

Giordano (2018) define a la morfología como el estudio de las formas caracterizado por el enfoque o punto de vista con el que se aborda este campo disciplinar, enfatizando en su concepción como "modo de pensar", esto explicaría los cambios en la noción de forma a través de la historia y el pensamiento y nos permitiría analizar las diversas producciones en contextos diferentes. Para la autora, la problemática de la morfología se explicaría desde un sistema de relaciones intrínsecas en donde se ponen en juego las relaciones entre geometría, significación, estructura formal y concreción material, en el marco de "lógicas relacionales". Enfatiza también en la comprensión de la forma como producto cultural, ámbito de valoración de su existencia. A estas lógicas relacionales, nos interesa mirarlas y /o contrastarlas con las lógicas de coherencia propuestas por Pokropek (2020).

Aquella idea de forma asociada a la apariencia de las cosas y a la disposición física fue superada, según Giordano (2018),a inicios del siglo XX, momento en que toma protagonismo el Diseño en el marco de teorías estructuralistas, aportes de la lingüística y de la semiótica. Por otra parte, señala que: 
En la morfología, las entidades geométricas no pueden ser consideradas neutras; son interpretadas desde los elementos que constituyen sus posibles estructuras. Las formas portan sentido en una cultura y así también el Diseño, operador de formas, no es un ejercicio neutro de resolución de problemas funcionales sino una práctica comprometida con el contexto social...la morfología podría plantearse como el estudio de los modos en que las distintas culturas comprenden la forma (Giordano, pág. 119)

En sintonía con estas afirmaciones, Montaner (2002) sostiene que la palabra forma es ambigua, en el sentido que depende del lugar de enunciación y por la cantidad de significados que se le atribuyen. Según el autor la forma nada tiene que ver con la silueta, apariencia exterior, contorno ni estilo. La noción que propone tiene que ver con "estructura esencial e interna, como construcción de espacio y materia" (pág. 8). La clave estaría en comprender a la noción de estructura como articulador de los diversos significados de forma, en donde forma y contenido podrían coincidir. Esta noción ya estaba presente en los postulados aristotélicos de estructura como esencia y en las categorías: substancia, cantidad, cualidad, relación, acción, pasión, cuándo, dónde, situación y hábito. Esta idea de forma/esencia fue retomada a comienzos del siglo XX con las vanguardias abstractas para posteriormente consolidarse con las teorías lingüísticas y el pensamiento estructuralista (Montaner, 2002).

Si se consigue demostrar que la infinita diversidad de formas son agrupables en un repertorio limitado de lógicas o mundos formales; que en cada uno de dichos mundos es posible detectar las raíces y las leyes que los rigen; que existen implicaciones entre las formas y las éticas; que anudadas en torno a dichos repertorios de formas existen teorías científicas, filosóficas, estéticas y política que los legitiman y que definen distintas condiciones de tiempo y del sujeto; que cada posición recurre a ciertas técnicas materiales y concepciones de la estructura constructiva, y que cada mundo formal se comporta de cierta manera en relación al lugar y al contexto (Montaner, 2002, pág. 12)

Importa así, descubrir las lógicas y mundos formales que responden al habitar campesino en las formas de la arquitectura vernácula, estrechamente vinculada al contexto. Para indagar sobre la noción de habitar, y articular el análisis con las prácticas sociales que lo definen, es indispensable referirnos a ésta como condición inherente al ser humano. Se dice que el ser humano es el único ser viviente que carece de hábitat natural o que ha renunciado a él, por lo que tiene que construirlo; esta construcción social de las espacialidades llamada vivienda, puede entenderse como una forma de habitar que evidencia los modos en que cada cultura entabla su relación con el entorno. Los modos como lo hace o las formas que produce son de interés para esta reflexión que se centra en cuestiones de morfología.

Todo ser humano, de manera individual o colectiva, deja una huella permanente de su actuar en construcciones sociales y materiales, entre éstas, la vivienda representa una de las formas visibles del habitar. Cada cultura a lo largo de la historia ha edificado sus viviendas, 
principalmente como una respuesta encaminada a la satisfacción de necesidades básicas y través de ellas ha puesto de manifiesto una particular manera o forma de vivir (Malo, 2012). Nos referiremos a "forma", no como entidad abstracta en una concepción desde la geometría, como disposición física, sino en sus posibilidades de interpretación a partir de comprenderla como una estructura y en este sentido, Giordano (2018) propone comprender esta estructura de la forma, a través de la integración de factores formales, funcionales y tecnológicos, incluido el contexto.

Giordano (2018) propone comprender a la morfología en base a la comprensión de la misma como un fenómeno cultural producido y generado por culturas concretas, ámbito de referencia y existencia, señala también que la significación es un rasgo intencionado en las lógicas del Diseño y que la noción de interpretación es factor clave en la comprensión e interpretación de la forma, así:

...la noción de interpretación es clave en la comprensión de las formas. La interpretación se opera a través de una estructura relacional entre significantes y significados". El significante es el lenguaje expresivo que caracteriza una forma o grupo de formas, el significado se plantea en términos de relaciones subyacentes en el modo de concebir y concretar una forma. Se podría decir que los significados guían la operatoria del Diseño, cuya operación se concreta en el campo de los significantes (Giordano, 2018, pág. 117).

Hablar de la vivienda popular campesina, supone comprender la cultura popular, al sujeto que habita, las prácticas sociales y analizar las estructuras que conforman esta compleja construcción social vinculada estrechamente a valores y significados que consolidan la identidad. Es en la vivienda y desde la vivienda, como el entorno más próximo del ser humano, en donde se hace posible que se definan aspectos esenciales de la vida en sociedad, como son: valores y principios, roles y costumbres, visiones y creencias, entre otros.

La reflexión y análisis sobre la morfología de la vivienda campesina intenta esclarecer algunas de las realidades que configuran la espacialidad como noción de forma habitable en el marco de prácticas sociales que son referentes en la conformación de un espacio y que, como significados y valores "dan forma” al habitar en un sentido metafórico y real.

Nos interesa analizar y comprender la morfología de la vivienda campesina inmersa en la problemática del habitar rural - habitar campesino, como un entramado de relaciones que se establecen entre la estructura familiar, el entorno, la producción, la tierra, las tradiciones, las creencias y la comunidad.

Todo ser humano, de manera individual o colectiva, deja una huella permanente de su actuar en construcciones sociales y materiales, entre éstas, la vivienda representa la forma más visible del habitar. Cada cultura a lo largo de la historia ha edificado sus viviendas, principalmente como una respuesta encaminada a la satisfacción de necesidades básicas y través de ellas ha puesto de manifiesto una particular manera de vivir. 


\section{Análisis de las formas de habitar y formas para habitar en las viviendas campesinas}

Para abordar la comprensión de la problemática de la forma, se propone el siguiente esquema a manera de estructura relacional, no como estructura fija sino como planteo de relaciones posibles que sugieren conjeturas parciales en el campo disciplinar (Giordano, 2018). El siguiente esquema da cuenta de una construcción de sentido a través de problematizar relaciones que no estarían solamente en el sujeto - objeto sino en relación al contexto y posicionamiento.

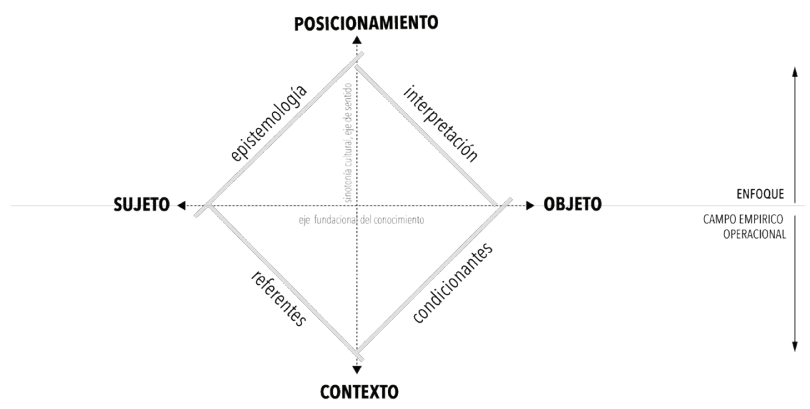

Imagen 1.

El gráfico refiere a un esquema relacional que ubica en la parte superior al enfoque y en la parte inferior hace referencia al campo empírico.

El posicionamiento señala, en el gráfico, el marco referencial teórico, es decir el lugar desde donde hablamos y las teorías que se enuncian, en este estudio nos interesa poner en relación los criterios morfológicos de lógicas de coherencia de Jorge Pokropek con un enfoque interpretativo de la morfología a partir de reflexiones de Montaner (2002) y Giordano (2018). El sujeto: en este caso particular de análisis, vinculado a formas del habitar, nos referimos al sujeto que habita el campo del Azuay y Cañar (el campesino, en nuestro análisis).

El objeto: es el objeto de análisis, el objeto de estudio en esta reflexión y refiere, en este caso a la vivienda como objeto, como forma, como espacio.

El contexto: hace referencia a las condiciones culturales, ambientales y sociales en donde se inserta el objeto de estudio. Para este caso de análisis serán las condiciones de paisaje como entorno inmediato, la cultura y las prácticas de vida nociones del habitar campesino.

\section{Relación objeto - contexto como configurador de las categorías estructu- rales y texturales}

Se propone, en este análisis, indagar sobre las articulaciones entre las lógicas relacionales de la forma (Imagen 1) con las categorías estructurales de la forma: formas arquitectónicas y 
estructuras abstractas; lógicas de configuración y lógicas de organización (Pokropek, 2020). En el siguiente gráfico, entendemos a la vivienda campesina como nuestro objeto de estudio. En contexto ubicamos al conjunto de prácticas sociales ejercidas y reconocidas por un determinado grupo social, para nuestro caso de estudio referido a la vivienda campesina, comprendemos que existen prácticas sociales validadas comunitariamente y que reflejan una forma de habitar referida al contexto en cuestión. "La vida en el campo en nuestra región, ha estado siempre muy marcada por contexto, vivir en el campo supone aceptar las reglas que esta condición impone: la faena diaria, el estrecho vínculo con la tierra, la austeridad y simpleza, la dependencia familiar y comunitaria” (Malo, 2012, pág. 183)

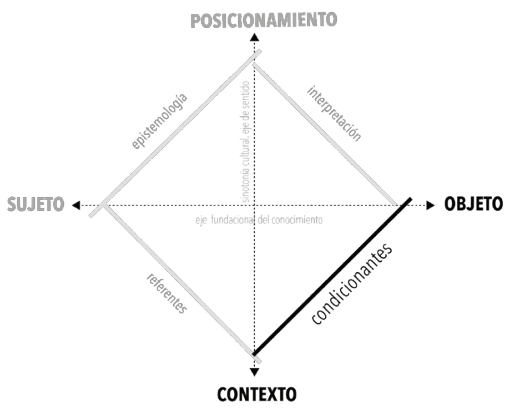

Imagen 2.

Entre objeto y contexto (ver Imagen 2) proponemos ubicar los condicionantes del habitar: son los usos, los usuarios y las actividades que, con sus características, condicionan un modelo de prácticas reconocidas por un grupo humano que habita en el campo y ha definido a lo largo de la historia una manera de vivir. Este conjunto de prácticas refleja un momento primario de construcción del habitar rural que construye su vivienda en base a usos, usuarios y actividades.

Características de uso de la vivienda campesina: Los usos de la vivienda campesina se comprenden, inicialmente, desde la satisfacción de necesidades básicas: la protección, el cobijo, el alimento; y, como condición ineludible, el trabajo en el campo, pues a partir de esta actividad es que se construye la vivienda y su significado. Las prácticas sociales estrechamente vinculadas a este uso tienen que ver con lo cotidiano, con los actos individuales y grupales que se desarrollan en espacios interiores y exteriores de la vivienda: espacios para guardar, preparar e ingerir alimentos, un espacio para descansar y un espacio para socializar. A partir de estas consideraciones, la vivienda se estructura en zona de servicio, zona de descanso y zona social.

Características de los usuarios de la vivienda campesina: En la modesta vivienda del campo los usuarios son la familia y muy comúnmente también los animales. En el campo, 
viven y trabajan padres e hijos, con tareas asignadas que dependen de la edad y el sexo; son las labores diarias, tarea de todos, las que permiten el sustento de la familia. El concepto primario de comunidad se construye en su célula inicial, la familia campesina.

Actividades al interior de la vivienda campesina: tienen que ver principalmente con la satisfacción de necesidades básicas: principalmente la preparación y consumo de alimentos, el descanso, la protección, la estrecha socialización familiar, el almacenaje de la producción agrícola, herramientas para el trabajo y el cuidado de animales. La vivienda se estructura en base a estas actividades principales y, de alguna manera, se muestra fragmentada en tres áreas principales: servicio (cocina), descanso (dormitorio) y social (soportal), estos espacios están divididos por paredes dentro de un mismo bloque y en otras ocasiones se conforman volúmenes independientes.

A partir estas formas de vida suponemos que se genera una estructura inicial, una matriz primaria de la forma, unas categorías estructurales, que al decir de Pokropek (2020) "nos referimos a una matriz inmaterial que organiza las formas, conceptualizable también como ley rectora que establece la coherencia armónica o ligazón entre las partes de un sistema” (pág. 21). Geometría que a manera de lógica de organización reúne y separa líneas, puntos y áreas con una clara intención configurativa.

Una inicial conformación de estas formas, de acuerdo a Pokropek (2020) podría leerse en siete categorías de pares y en el principio de orden que rige una estructura: si es fuerte y evidente o difusa o débil. Es decir, en una primera clasificación serían las estructuras definidas y ordenadas frente a las indefinidas o aleatorias como se observa en el gráfico a continuación.

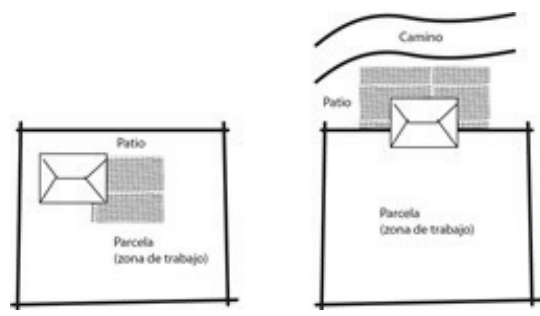

Imagen 3.

Una matriz inicial de la forma de la vivienda campesina, muestra en el gráfico, la manera en que ésta establece relaciones con el entorno inmediato (las parcelas de trabajo) en donde se emplaza la vivienda.

En un segundo momento de análisis de Pokropek (2020) habla del grado en que las estructuras se manifiestan: limitadas o ilimitadas, unitarias o múltiples, estáticas o dinámicas, quietas o móviles, rígidas o elásticas, sólidas o articuladas, focalizadas o difusas.

Podríamos definir observar que, entre las 7 categorías estructurales mencionadas anteriormente, en la vivienda campesina prevalecen las siguientes categorías si las analizamos por 
unidades: Limitadas, unitarias, múltiples, estáticas, quietas, rígidas, sólidas, articuladas y focalizadas. En cambio si las analizamos en relación de pares, prevalecen las relaciones: unitario/múltiple, sólido/articulado no en oposición sino en relación. Así establecemos la presencia de algunas lógicas relacionales y lógicas de coherencia, como observamos a continuación:

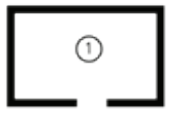

Caso 1: Vivienda Popula rural. Un solo espacio

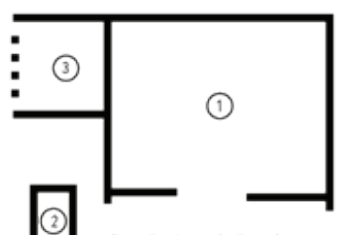

Caso 3: Vivienda Popular cocina externa 3 espacios 1) vivienda 2)cocina 3)pesebre

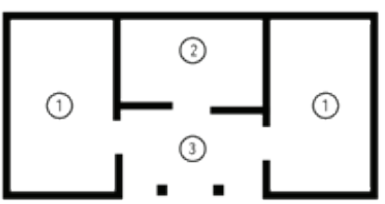

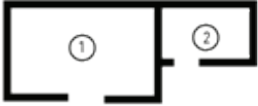

Caso 2: Vivienda Popula

rural. Dos espacios

1) viviend.

2)cocina
Imagen 4.

Fuente: Muñoz Vega,

2015, pág. 75

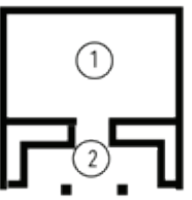

Caso 4: Se adiciona el espacio semi público

3 espacios:

1)vivienda

2) corredor con poyos

3)cocina exenta

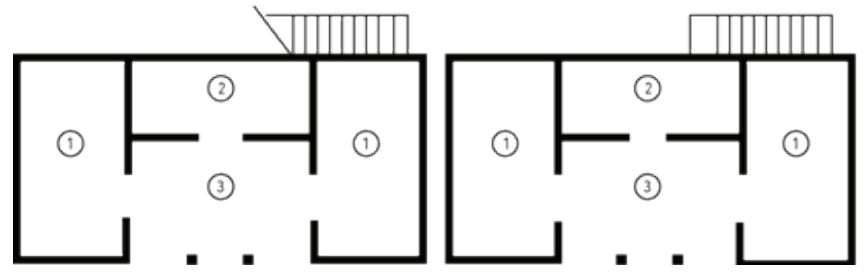


Por otro lado, de acuerdo a lo expuesto por Pokropek (2020) es importante recordar que la forma arquitectónica posee al menos dos tipos de estructura abstracta: la envolvente o volumetría y la espacialidad o interior que deberían prefigurarse por separado por constituir fenómenos perceptuales distintos. En nuestro caso de estudio podemos identificar características de la espacialidad similares a la envolvente, casi como una consecuencia; sin embargo, las relaciones que se establecen y que tienen que ver con los modos de vida, son diferentes, las relaciones público - privado son muy definidas corresponden casi unívocamente a la relación envolvente - espacialidad. Hacia afuera lo público, hacia adentro lo privado en el más estricto sentido.

En la envolvente o volumetría de la vivienda campesina identificamos las relaciones sólido/vacío principalmente en lo que se llama "el soportal", espacio característico de la vivienda rural en la sierra, un lugar semiabierto y por lo tanto semiprivado, en donde se realizan diversas actividades como descansar y mirar, recibir visitas, realizar artesanías, guardar herramientas, juego de niños y en ocasiones hasta comer.
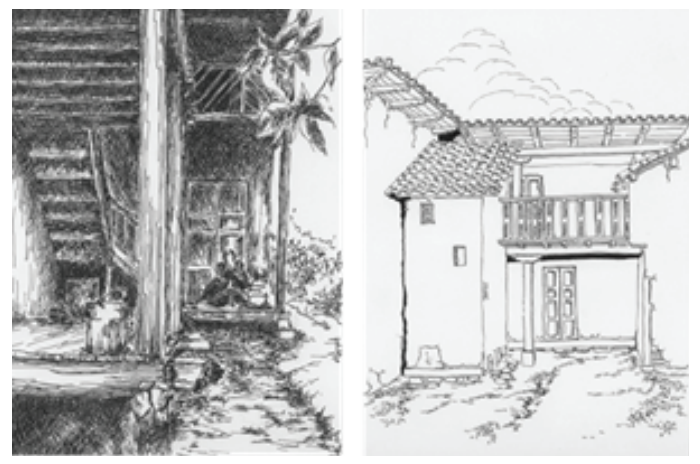

Imagen 5 .

Viviendas Azuay-Chordeleg. Fuente: Muñoz Vega, 2015.

La vida en comunidad es actividad importante, que influye en la forma de la vivienda campesina y justifica la presencia del patio junto a la vivienda. El campesino tiene arraigado en sus costumbres y su manera de vivir, un profundo sentido de solidaridad y pertenencia comunitaria, vive y opera como miembro de una comunidad. Los festejos, las celebraciones, los momentos de alegría, así como los de dolor, son compartidos con los vecinos y los miembros de la comunidad, que constituyen una gran familia, el patio y el soportal son los lugares para compartir con la comunidad.

Vivir y trabajar en el campo implica seguir las normas de la naturaleza: los ciclos, tiempos, leyes y reglas que solo quien habita en el campo las conoce; es a partir de esta reflexión que situamos otro de los niveles de la teoría del habitar, una manera habitar que se justifica por el contexto más cercano, el contexto natural y sus condiciones. 
Las categorías texturales, según Popropek (2020) pueden ser explicitas o implícitas, según la materialidad o inmaterialidad que las caracteriza, de modo que:

Para su percepción sensible requieren de una materialidad visible que expresa ciertas características formales. Esta expresión de la materialidad sensiblemente percibible recibe el nombre de textura o mostración superficial. Ras dice que se puede definir la textura como la modalidad según la cual se pone de manifesto la superficie o volumen de un objeto (pág. 22)

En la vivienda campesina, las formas texturales adquieren una característica que revela un alto grado de conexión con el entorno(ver imagen (Tamayo \& otros, Arquitectura Vernácula en Azuay y Cañar, 2017)), de modo que casi se mimetizan pues las texturas (superficies de los volúmenes), de los materiales extraídos de la propia tierra se expresan en sus rasgos formales de color y textura, como es el caso del adobe, fabricado con material del entorno, la madera de bosques cercanos, el ladrillo, la paja, la piedra, entre otros materiales que son la base fundamental para la fabricación de este.

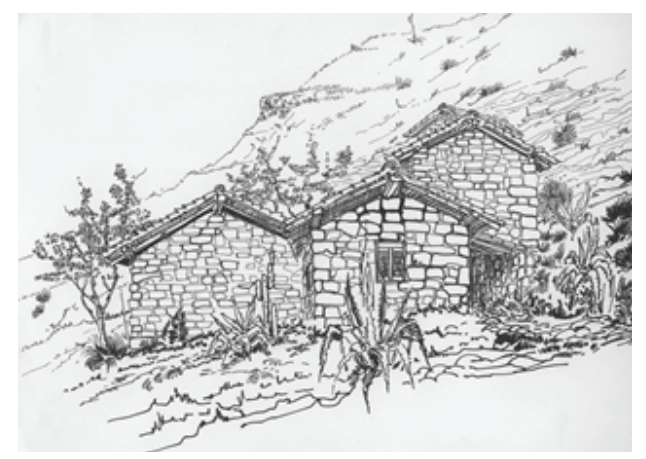

Imagen 6.

Viviendas Azuay-Chordeleg. Fuente: Muñoz Vega, 2015.

En la investigación realizada (Arquitectura Vernácula en Azuay y Cañar) se puede comprobar que la gran mayoría de los materiales utilizados son provenientes del entorno y en consecuencia otorgan texturas en relación casi de mímesis con el entorno.

En este sentido la relación de la categoría textural de superficie del objeto/vivienda en relación al espacio circundante es de completa correspondencia con los materiales del contexto. 


\begin{tabular}{|c|c|c|c|c|}
\hline \multirow{2}{*}{\multicolumn{2}{|c|}{ Variables }} & \multirow{2}{*}{$\begin{array}{c}\text { Azuay } \\
N=594\end{array}$} & \multirow{2}{*}{$\begin{array}{l}\text { Cañar } \\
\mathrm{N}=409\end{array}$} & \multirow{2}{*}{$\begin{array}{c}\text { Total } \\
\mathrm{N}=1003\end{array}$} \\
\hline & & & & \\
\hline \multirow{3}{*}{ Emplazamiento } & aislada & 246 & 197 & 443 \\
\hline & continua & 165 & 99 & 264 \\
\hline & pareada & 179 & 113 & 292 \\
\hline \multirow{2}{*}{ Entorno } & agrícola & 283 & 208 & 491 \\
\hline & edificado & 308 & 201 & 509 \\
\hline \multirow{4}{*}{ Número de pisos } & 1 & 232 & 138 & 370 \\
\hline & 2 & 338 & 250 & 588 \\
\hline & 3 & 21 & 21 & 42 \\
\hline & más de 3 & 3 & 0 & 3 \\
\hline \multirow{2}{*}{ Cimentación } & piedra & 577 & 388 & 965 \\
\hline & otro & 2 & 18 & 20 \\
\hline Sobre cimiento & $\mathrm{SI}$ & 437 & 322 & 759 \\
\hline \multirow{2}{*}{ Columnas } & madera & 526 & 383 & 909 \\
\hline & otro & 29 & 15 & 44 \\
\hline \multirow{2}{*}{ Vigas } & madera & 567 & 393 & 960 \\
\hline & otro & 9 & 8 & 17 \\
\hline \multirow{5}{*}{ Muros } & adobe & 367 & 96 & 463 \\
\hline & bahareque & 163 & 239 & 402 \\
\hline & tapial & 10 & 4 & 14 \\
\hline & madera & 11 & 7 & 18 \\
\hline & otro & 27 & 63 & 90 \\
\hline \multirow{3}{*}{ Cubierta } & teja & 550 & 304 & 854 \\
\hline & zinc & 16 & 55 & 71 \\
\hline & otro & 22 & 49 & 71 \\
\hline \multirow{4}{*}{ Pisos } & piedra & 16 & 28 & 44 \\
\hline & tierra & 341 & 267 & 608 \\
\hline & madera & 94 & 46 & 140 \\
\hline & ladrillo & 106 & 19 & 125 \\
\hline Puertas & madera-.... & 586 & 405 & 991 \\
\hline Ventanas & madera.... & 520 & 358 & 878 \\
\hline Balcones/balaustrada & $\ldots$ & 230 & 197 & 427 \\
\hline
\end{tabular}

Imagen 7. Fuente: (Tamayo \& otros, 2017) 
Entre categorías formales/texturales y estructurales es posible también analizar configuraciones arquitectónicas en relaciones que se ponen en juego: homogéneo-heterogéneo, denso diáfano, transparente-opaco, esquemático-farragoso, céldico-ambitual, luminosooscuro, brilloso-mate, permeable-impermeable (Pokropek, 2020).

Según Pokropek (2020) las categorías texturales estructurales permiten establecer patrones en las relaciones forma-conducta, forma-emoción. Para la vivienda, motivo de nuestro análisis, es evidente que las estructuras céldicas - ambituales generan el recogimiento y estructura de hogar propuesta desde las prácticas de vida descritas en párrafos anteriores. Los patrones de conducta y usos de espacios en la relación público privado se reafirman con las categorías estructurales más utilizadas.

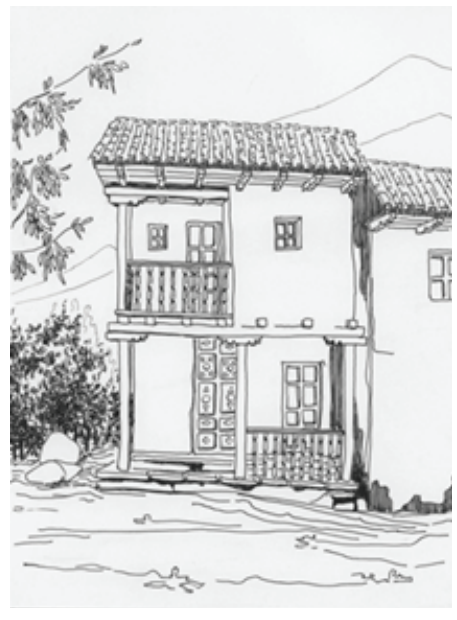

Imagen 8.

Estructura céldica - ambitual

Se encuentra también la categoría heterogéneo homogéneo, donde prevalece lo homogéneo y la categoría denso-diáfano con equilibrio, como se observa en la imagen.

\section{Relaciones contexto - sujeto como configurador de las cadenas armónicas}

En este cruce se ahonda en la relación que podría tener el sujeto/habitante campesino en relación al contexto de prácticas y cómo esta dinámica podría configurar las cadenas armónicas. En el análisis que planteamos, consideramos al contexto como el espacio primario en el que se inserta la cultura campesina en sus orígenes y explica la configuración de un habitar fuertemente ligado y condicionado al contexto. 


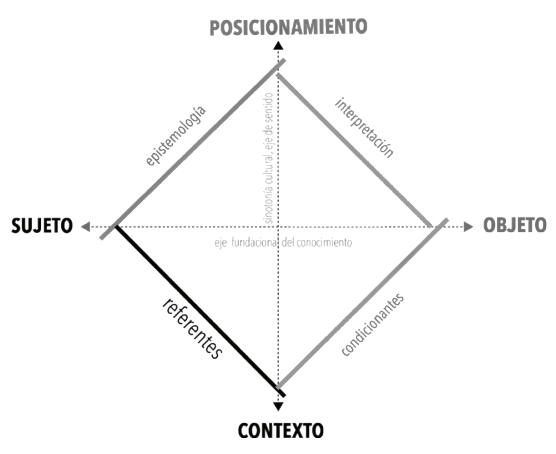

Imagen 9.

Para comprender el caso de la vivienda campesina, esta relación puede abordarse desde reflexiones antropológicas, sociológicas o histórico-culturales que dan cuenta de la existencia de las normas construidas entre la necesidad y la realidad; y que, han sido validadas en el tiempo, pero sobre todo nos interesa reflexionar sobre las relaciones que se plantean entre los niveles; podríamos entender que en la relación sujeto-contexto estarían los referentes. Como referentes del contexto primario de la vivienda campesina, situamos a:

- El sitio: podemos entenderlo a partir de la relación con la tierra, y la parcela como elemento de pertenencia y supervivencia. El trabajo principal del campesino está en la agricultura, la ganadería y cualquier práctica derivada de estas actividades, como venta, intercambio de productos y elaboración de artesanías. Por lo tanto, la vivienda campesina responde a esta estrecha relación de dependencia y su implantación junto o dentro de la parcela de trabajo es una respuesta a esta relación.

- El contexto: La proximidad a la tierra, a la vegetación, a los cultivos, a los animales marca una manera de vivir en el campo que se caracteriza por responder de un modo simple, austero y eficiente a las necesidades de la de una vida cercana a la naturaleza, la manera como se estructura la vivienda es un reflejo este tipo de vida y ha sido ajena, en general, a los nuevos sistemas de comodidad y desarrollo de la vida de las nuevas sociedades urbanas. Habitar en el campo implica hacerlo suyo, permanecer, respetar y vivir bajo sus normas.

- El clima: en la sierra austral del país el clima es templado y frío, con fuertes y variadas precipitaciones, condiciones que han influido fuertemente en el tipo de construcción que busca principalmente protección y abrigo. El acondicionamiento ambiental al interior de las viviendas, se resuelve medianamente en lo que respecta a climatización, por las bondades de los materiales utilizados como el adobe, que tiene propiedades térmicas; sin embargo, la iluminación y ventilación es deficiente puesto que los ingresos de aire y luz son limitados a áreas muy reducidas de ventanas. 
Usos, usuarios y actividades en la vivienda campesina se justifican como prácticas sociales por la existencia de un sistema de relaciones que el hombre ha entablado en con el entorno natural y que responden además a las condiciones ya señaladas de sitio, contexto y clima. Las cadenas armónicas definidas como conjunto de polaridades puestas en juego, tienden a fortalecer la coherencia interna debido a que la presencia en simultáneo permite el mutuo refuerzo de las características y por lo tanto asegura el nivel de autorreferencialidad, como premisa del mensaje estético (Pokropek, 2020).

$\mathrm{Si}$ analizamos los condicionantes que pueden emerger de relación sujeto - contexto: sitio, paisaje y clima, podemos encontrar relaciones importantes entre éstos y las configuraciones o lecturas de las formas (forma-vivienda). En este sentido son fuertes estímulos para las configuraciones arquitectónicas.

El proceso de construcción de la vivienda no solo constituye una de las actividades de la vida del hombre campesino, que entabla una fuerte relación con el entorno y con la comunidad. Los procesos participativos en la construcción, el uso de los materiales de la tierra, dan cuenta de estos vínculos.

\section{Relación objeto -sujeto, mediado por el contexto como configurador de las categorías estructurales y texturales}

Como un producto de la relación entre sujeto y objeto, mediada por la cultura, se entiende el complejo entramado del habitar y, de igual manera que en el habla, los signos se construyen entre significados y significantes. Son las viviendas campesinas "formas" para habitar, son significantes sistematizados en tipologías constructivas y morfológicas que se validan en el uso como signos de identidad que han representado en nuestra región una forma de asumir la vida en el campo.

Por otro lado, los tipos configurativos básicos que se conceptualizan desde un enfoque fenomenológico, según Pokropek (2020) son 4:

- Los recintos o habitaciones: tendientes a estimular en el usuario conductas de permanencia o quietud, generalmente los espacios configurados son estáticos, que pueden tener conectores entre sitios de llegada y permanencia.

- El 'sostén' de figuras plásticas: que promueve el desplazamiento y movimientos exploratorios y se opone al anterior.

- El tipo configurativo espacial básico: que consiste en la 'partición de lo continuo' caracterizado por la negación de la vocación recintual y el estímulo para la fluidez de movimiento.

- La fusión de lo continuo: similar al anterior, pero se agrega la sensación de vacíos intersticiales, son propuestas más radicales como las de Zaha Hadid o Rem Koolhaas. 
Resulta interesante analizar estos tipos configurativos básicos en el marco de las viviendas campesinas, construidas principalmente con volúmenes y cómo éstos estimulan respuestas conducto emocionales, asociadas a las prácticas sociales y formas de vida. Diremos entonces que, estos signos construidos, se evidencian en construcciones materiales y sociales que responden un esquema de relaciones, de la que surgen las configuraciones espaciales.

Entre usos y sitio: emergen configuraciones espaciales como formas de emplazamiento y como formas de vivienda
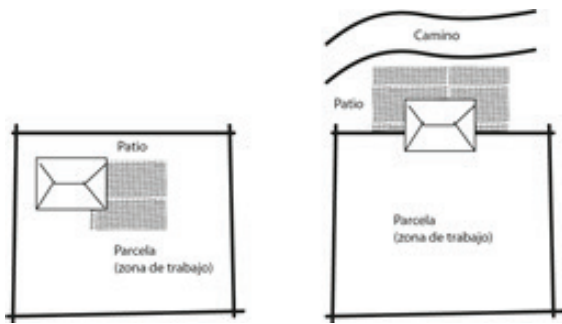

Imagen 10.

Los diversos tipos de implantación, ponen de manifiesto las consideraciones sobre las relaciones del campesino con vivienda. La relación interior-exterior es muy marcada y definida por estructuras y usos.

Tipologías de organización espacial como recintos o habitaciones con conectores entre zonas de llegada y permanencia.

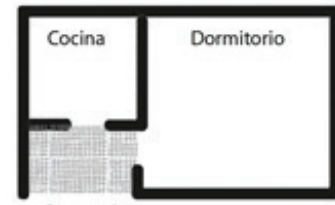

Soportal

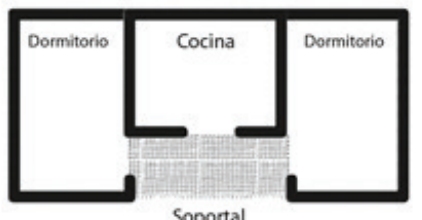

Imagen 11.
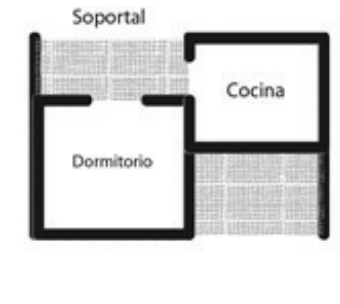

La manera como se organiza el espacio está dada principalmente por la contribución de la vivienda en apoyo al trabajo. En las viviendas de un solo bloque, generalmente se antepone el patio a la vivienda, constituyéndose éste en el acceso principal. La parte posterior en cambio, se conecta con la parcela y por ende con el trabajo, si en la vivienda existe sitio para almacenar las herramientas de trabajo, estaría ubicado en este lugar. 
El patio existe en casi la totalidad de las viviendas campesinas, es el espacio destinado al juego de los niños, a la recepción de visitas (puede también hacerse en el soportal), y en algunos casos al almacenaje de granos y herramientas. La presencia de los animales es una importante característica.

Existen algunos tipos de patios, que cumplen diversas funciones (separar, articular, configurar un acceso, entre otras), pueden ser además abiertos (sin cerramiento) o cerrados (enmarcados en muros, generalmente de piedra).

Existen también viviendas que se conforman en dos o más bloques, generalmente articuladas por un patio central. Podríamos encontrar el mismo tipo configurativo anterior (recintual) con conectores entre
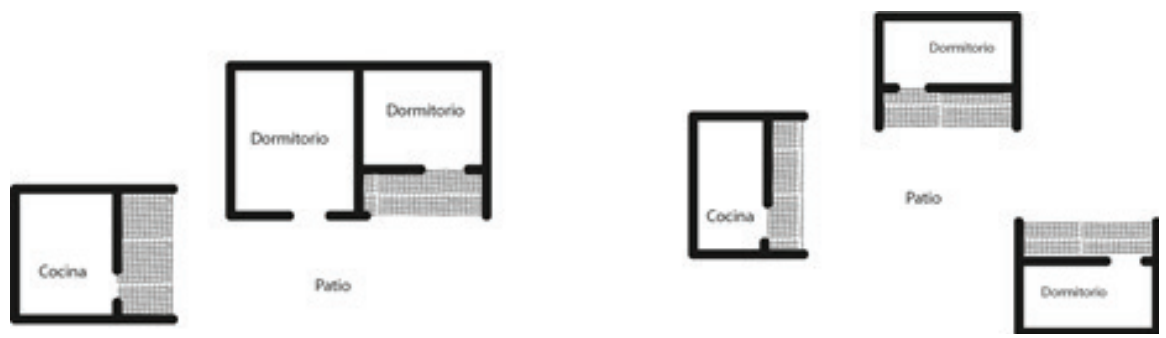

Imagen 12.

Estos modos en que se emplazan y organizan las viviendas ponen en evidencia consideraciones culturales frente a lo público y lo privado. Para el campesino del austro, el patio y el soportal son generalmente los lugares públicos, la cocina puede considerarse un espacio semi-privado y los dormitorios un espacio privado.

El patio está presente en casi todas las viviendas campesinas y es un elemento articulador con diversos bloques, con el camino o con otras parcelas; el patio puede ser central, irregular, cerrado o abierto.
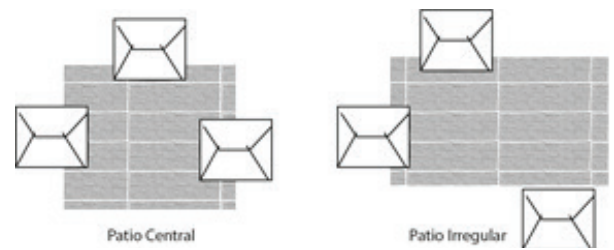

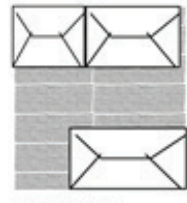

Patio Cerrado

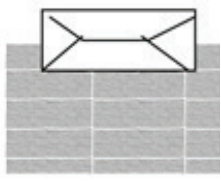

Patio Abierto

Imagen 13.

Las formas espaciales y articulaciones son también el reflejo de una cultura basada en la austeridad y la simplicidad. 


\title{
Conclusiones:
}

El breve análisis y reflexión propuesta da cuenta de una manera de comprender la morfología de la vivienda como un sistema de relaciones que puede ser observada desde categorías estructurales y formales en vinculación al contexto que la genera, la produce y da sentido. Así, la forma se construye como producto cultural.

Podría entenderse que, de esta relación con el contexto, surgen las tipologías constructivas y el uso reiterativo de materiales como el adobe, el tapial y el bahareque, estas técnicas y su particular manera de utilizarlas son parte de la forma de la vivienda.

\section{Listas de Referencias Bibliográficas}

Doberti, R. (2012). De la descripción de las costumbres a la teoría del habitar. Buenos Aires: Ediciones FADU-UBA.

Doberti, R. (2008). Espacialidades. Buenos Aires: Ediciones Infinito.

Giordano, D. (2018). Cuestiones de Diseño, equilibrios inestables sobre campos imprecisos. Buenos Aires: Edición diseño editorial.

ICOMOS. (1999). Carta del Patrimonio Vernáculo Construido. Recuperado el Noviembre de 2018, de http://www.icomos.org/charters/vernacular_sp.pdf.

Malo, G. (2012). Vivienda Popular. Universidad Verdad, 179-194.

Montaner, J. M. (2002). Las formas del siglo XX. Barcelona: Gustavo Gili.

Muñoz Vega, P. (2015). Arquitectura popular en Azuay y Cañar. Cuenca: Univerisidad de Cuenca - CIDAP.

Pokropek, J. (2020). Lógicas de coherencia para la interpretación y produccón del diseño interior y sus criterios de selección de formas objetuales. Cuaderno (81), 19-29.

Rappoport, A. (1969). Vivienda y Cultura. Barcelona: Gustavo Gili.

Tamayo, J., \& otros, y. (2017). Arquitectura Vernácula en Azuay y Cañar. Cuenca: en prensa.

Tamayo, J., Malo, G., \& García, G. (aprobada para publicación 2019). El dibujo y su aporte a la identificación de valores de la Arquitectura Vernácula. ESTOA .

Zorrila, H. (2015). El concepto de Arquitectura Vernácula. Recuperado en diciembre de 2018, de http://arquiecturadecasas.info/el-concepto-aquitectura-vernácula/.

\begin{abstract}
This paper proposes an analysis of the referents and conditions that constitute popular peasant housing in the Ecuadorian provinces of Azuay and Cañar, as an expression and form of vernacular architecture. The study begins by problematizing the system of rural living. Spatial configurations are described and analyzed in the framework of a conceptual scheme that takes context as a node to articulate meaning and analysis, based on reflections by Jorge Pokropek (2020), who raises the relationship between social practices and spatial configurations. This relationship carries meaning and identity to define logics of coherence as a mechanism of interpretation and production of interior design. It also brings the notion of form proposed by Dora Giordano (2018) as a cultural product,
\end{abstract}


and Joseph María Montaner who proposes to understand the form as a structure, beyond the geometric configuration. The reflections and analysis on the subject that is exposed are based on data obtained in a research carried out between the University of Cuenca and the University of Azuay in 2016. The data consists of data itself and drawings by hand -pen nibs and observation cards- of the vernacular architecture between 1977 and 1978 in the provinces of Azuay and Cañar.

Keywords: Ways of inhabit - notion of form - house morphology - peasant housing vernacular architecture

Resumo: Este artigo propõe a análise dos parâmetros e condições que moldam a habitação rural nas províncias equatorianas de Azuay e Cañar, como expressão e forma da arquitetura vernácula, desde a problematização do sistema do habitar rural. Se descreve e analisa configurações espaciais dentro de um quadro conceptual que toma o contexto como núcleo articulador de sentido e análise, a partir de reflexões de Jorge Pocropek (2020), que coloca a relação entre práticas sociais e as configurações espaciais que portam sentido e identidade para definir lógicas de coerência como mecanismo de interpretação e produção de design interior, como também a noção de forma proposta por Dora Giordano (2018) como produto cultural, e Joseph María Montaner que propõe compreender à forma como uma estrutura, além da configuração geométrica. As reflexões e análise sobre o tema que se expõe sustentam-se em dados obtidos numa pesquisa feita entre a Universidade de Cuenca e a Universidade do Azuay em 2016 que toma dados e desenhos feitos a mão (plumas e fichas de observação) da arquitetura vernácula entre os anos 1977 e 1978 nas províncias de Azuay e Cañar.

Palavras chave: Modos de habitar - noção de forma - morfologia da habitação - habitação camponesa- arquitetura vernácula

[Las traducciones de los abstracts fueron supervisadas por el autor de cada artículo] 